\title{
Supplementary Information for Paper: Entanglement Transition of Randomly Branched Polymers in the Hyperbranched Class
}

June 23, 2006

\section{Details of the reaction-kinetics model}

The reaction system contains three different types of monomer, para-difunctional $(\mathrm{P})$, meta-difunctional $(\mathrm{M})$ (each containing one A group and one B group) and trifunctional $(\mathrm{T})$, which contains two $\mathrm{B}$ groups. These groups are present with number fractions $f_{P}, f_{M}$ and $f_{T}$ such that $f_{P}+f_{M}+f_{\mathrm{T}}=1$, and with total initial monomer concentration $c_{0}$.

We proceed with the assumption that all A groups behave identically, but that $\mathrm{B}$ groups on different monomers react at different rates (we shall comment on the outcome of other possible assumptions below). We make the further assumption that the two $\mathrm{B}$ groups on a trifunctional $(\mathrm{T})$ monomer react independently, so that the reaction rate of each $\mathrm{B}$ group is unaffected by reaction of the other $\mathrm{B}$ group. This latter assumption is supported by NMR measurements of the degree of branching (DB) of HBP samples formed purely from $\mathrm{AB}_{2}$ monomers similar to the $T$ monomers. ${ }^{1,2} \mathrm{~A}$ value of $\mathrm{DB}=0.5$ is found which implies that the reaction rates of the two B groups on the T monomer are independent. ${ }^{3}$

Under these assumptions, we have three reaction equations, of form:

$$
\mathrm{A}+\mathrm{B}_{I} \rightarrow-\mathrm{AB}_{I}-\quad k_{I}
$$

where $I=\mathrm{M}, \mathrm{P}$ or $\mathrm{T}$ and $\mathrm{B}_{I}$ denotes a $\mathrm{B}$ group on monomer type $I$. Letting $p_{\mathrm{B}_{I}}$ be the probability that such a group has reacted, then standard second-order reaction kinetics gives rise to three rate equations, each of form

$$
\frac{d p_{\mathrm{B}_{I}}}{d t}=c_{0} k_{I}\left(1-p_{\mathrm{A}}\right)\left(1-p_{\mathrm{B}_{I}}\right)
$$

where $p_{\mathrm{A}}$ is the probability that an $\mathrm{A}$ unit on any given monomer has reacted. This is given by

$$
p_{\mathrm{A}}=f_{M} p_{\mathrm{B}_{M}}+f_{P} p_{\mathrm{B}_{P}}+2 f_{T} p_{\mathrm{B}_{T}}
$$


the factor of 2 arising because a $\mathrm{T}$ monomer contains two $\mathrm{B}$ groups. We have the constraint that $p_{\mathrm{A}} \leq 1$, with equality occurring at complete conversion.

By dividing one rate equation (2) by another, we obtain equations of form

$$
\frac{d p_{\mathrm{B}_{M}}}{d p_{\mathrm{B}_{T}}}=\frac{k_{\mathrm{M}}\left(1-p_{\mathrm{B}_{M}}\right)}{k_{\mathrm{T}}\left(1-p_{\mathrm{B}_{T}}\right)}
$$

which solve to give:

$$
\left(1-p_{\mathrm{B}_{P}}\right)=\left(1-p_{\mathrm{B}_{\mathrm{M}}}\right)^{\frac{k_{P}}{k_{M}}}=\left(1-p_{\mathrm{B}_{\mathrm{T}}}\right)^{\frac{k_{P}}{k_{T}}} .
$$

The whole reaction ensemble is thus determined by the following parameters: the number fractions $f_{M}, f_{P}$ and $f_{T}$ of monomers present in the initial mixture; two ratios of reaction rate constants $\left(\frac{k_{M}}{k_{P}}\right.$ and $\left.\frac{k_{T}}{k_{P}}\right)$, which to a first approximation should be held constant for a given chemistry, and one independent reaction probability, $p_{\mathrm{B}_{P}}$ say, which is related to the degree of conversion. For a given sample, $p_{\mathrm{B}_{P}}$ can be determined by matching the sample molecular weight.

\subsection{Concentrations of reacted groups}

For the $\mathrm{H} 10$ series, it was possible to measure relative concentrations of $-\mathrm{A}_{I} \mathrm{~B}_{J^{-}}$ reacted groups where $I$ and $J$ can both be $\mathrm{M}, \mathrm{P}$ or $\mathrm{T}$. Within the model outlined above, these are prediced to be, for $J=\mathrm{M}$ or $\mathrm{P}$

$$
\frac{\left[\mathrm{A}_{I} \mathrm{~B}_{J}\right]}{[\mathrm{AB}]}=\frac{f_{I} f_{J} p_{\mathrm{B}_{J}}}{p_{\mathrm{A}}}
$$

and for $J=\mathrm{T}$

$$
\frac{\left[\mathrm{A}_{I} \mathrm{~B}_{J}\right]}{[\mathrm{AB}]}=2 \frac{f_{I} f_{T} p_{\mathrm{B}_{T}}}{p_{\mathrm{A}}} .
$$

\subsection{Mapping onto linear, branched and terminal monomers}

Under the assumption that all A units react identically, the model has the property that, given that a B unit has reacted, the type of monomer it has reacted to is statistically independent of the kind of monomer the B unit is on. Under these conditions, one can map from $\mathrm{M}, \mathrm{P}$ and $\mathrm{T}$ monomers to monomers which are linear (L), with one B group reacted, branched $(\mathrm{Br})$ with two $\mathrm{B}$ groups reacted, and terminal (Te), with no B groups reacted. Choosing an A unit at random (which is what a B unit does when it reacts), the probabilities that this A unit is on a linear, branched or terminal monomer are:

$$
\begin{aligned}
p_{\mathrm{L}} & =f_{M} p_{\mathrm{B}_{M}}+f_{P} p_{\mathrm{B}_{P}}+2 f_{T} p_{\mathrm{B}_{T}}\left(1-p_{\mathrm{B}_{T}}\right) \\
p_{\mathrm{Br}} & =f_{T} p_{\mathrm{B}_{T}}^{2} \\
p_{\mathrm{Te}} & =f_{M}\left(1-p_{\mathrm{B}_{M}}\right)+f_{P}\left(1-p_{\mathrm{B}_{P}}\right)+f_{\mathrm{T}}\left(1-p_{\mathrm{B}_{T}}\right)^{2} .
\end{aligned}
$$

Note that $p_{\mathrm{L}}+p_{\mathrm{Br}}+p_{\mathrm{Te}}=1$. 


\subsection{Mapping onto "metallocene" parameters}

Read and McLeish ${ }^{4}$ demonstrated that, under certain reactor conditions (i.e. CSTR), the distribution of branched molecules in metallocene-catalyzed polyolefins could be characterised by just two independent parameters: a branching probability, $b_{\mathrm{U}}$ (the probability that a polymer strand chosen at random has a branchpoint in the "upstream" direction) and an average strand degree of polymerisation, $P_{x}$. Metallocene molecules have a statistical directionality (an "upstream" and "downstream" direction) that is matched by the chemical asymmetry of the present hyperbranched molecules, and a statistical self-similarity which is characteristic of the "mean field" description of hyperbanched polymers. ${ }^{5,6}$ In the limit of small $f_{T}$, and assuming this "mean field" description for the hyperbranched polymers (in particular, assuming no loops are formed), we can make a direct mapping onto the metallocene polymer distribution and obtain parameters $b_{\mathrm{U}}$ and $P_{x}$. Each hyperbranched molecule has a single unreacted A unit; the "upstream" direction points away from this unit and the "downstream" direction points towards it.

The monomer at the end of a linear strand is either branched or terminal. The branching probability, $b_{\mathrm{U}}$ is the probability that it is branched rather than terminal, i.e.

$$
b_{\mathrm{U}}=\frac{p_{\mathrm{Br}}}{p_{\mathrm{Br}}+p_{\mathrm{Te}}} .
$$

The probability that a linear strand within the molecule has degree of polymerisation $m$ (including the branched or terminal unit at the end of the strand) is

$$
p_{m}=p_{\mathrm{L}}^{m-1}\left(1-p_{\mathrm{L}}\right)
$$

giving an average strand degree of polymerisation of

$$
P_{x}=\frac{1}{1-p_{\mathrm{L}}} .
$$

\subsection{Number- and weight-average degree of polymerisation}

Defining $p_{n}$ to be the probability that a hyperbranched molecule attains degree of polymerisation $n$, we can write a generating function

$$
F(z)=\sum_{n=1}^{\infty} p_{n} z^{n} .
$$

Using the statistical self-similarity of the hyperbranched molecules, we can write

$$
F=z\left(p_{\mathrm{Te}}+p_{\mathrm{L}} F+p_{\mathrm{Br}} F^{2}\right)
$$


In terms of this generating function, the number- and weight-average degrees of polymerisation are obtained from

$$
\begin{aligned}
N_{\mathrm{n}} & =\left.\frac{\partial F}{\partial z}\right|_{z=1} \\
N_{\mathrm{w}} & =\frac{\left.\frac{\partial^{2} F}{\partial^{2} z}\right|_{z=1}}{\left.\frac{\partial F}{\partial z}\right|_{z=1}}+1 .
\end{aligned}
$$

By differentiating (14) and using the property $F(z=1)=1$, we obtain:

$$
\begin{aligned}
N_{\mathrm{n}} & =\frac{1}{\left(1-p_{\mathrm{L}}-2 p_{\mathrm{Br}}\right)} \\
N_{\mathrm{w}} & =2 \frac{p_{\mathrm{L}}+3 p_{\mathrm{Br}}-\left(p_{\mathrm{L}}+2 p_{\mathrm{Br}}\right)^{2}}{\left(1-p_{\mathrm{L}}-2 p_{\mathrm{Br}}\right)^{2}}+1 .
\end{aligned}
$$

\subsection{Comments on alternative assumptions}

The above model made the assumption that all A units behaved identically, but that $\mathrm{B}$ units on different monomers have different reactivities. This assumption appears to be reasonably consistent with the available NMR data for the H10 series. Moreover, predictions for $b_{U}$ and $P_{x}$ for the $\mathrm{H} 1$ series appear to be insensitive towards variations in reactivity of the $B$ units within the range consistent with the H10 NMR data.

We have tried the contrary assumption that the B units behave identically but that A units on different monomers have different reactivities. Under this assumption, one can write similar rate equations to eq. 2 and solutions of the form of eq. 4. One can also make a mapping onto linear, branched and terminal monomers, with the slight complication that the probability that a monomer is linear, branched or terminal is correlated with the probability that the A unit has reacted. Since there is only one monomer in each molecule with an unreacted A, this is not a severe complication. Comparing with the NMR data for the H10 series, we find that one can improve the description of these data by assuming that the $\mathrm{A}$ units have different reactivities, but that this improvement is not so marked as when we assume instead that the B units have different reactivities. With this alternative assumption, it remains the case that predictions for $b_{U}$ and $P_{x}$ for the H1 series appear to be insensitive towards variations in reactivity of the A units within the range consistent with the H10 NMR data.

It is likely that, in fact, all the A and B units have very slightly different reactivities. Description of this scenario is mathematically much more difficult. One would need to write individual rate equations of form (2) accounting for each A-B combination, with 9 separate rate constants. These do not have convenient solutions of form (4), but need to be integrated numerically for each combination of rate constant and monomer number fraction. In this general case, it is not possible to 
make the mapping onto linear, branched and terminal monomers because different kinds of $\mathrm{B}$ units react preferentially with different kinds of $\mathrm{A}$ unit. The form of probability distribution for the strand degree of polymerisation given in eq. 11 does not apply (although it is asymptotically of this form for long strands). In view of the apparent insensitivity of the parameters $b_{U}$ and $P_{x}$ to variations in reactivity where we have made calculations, we felt that little would be gained from a detailed and time-consuming examination of this general case. 


\section{References}

1. Feast, W. J.; Keeney, A. J.; Kenwright, A. M.; Parker, D. Chemical Communications 1997, 18, 1749-1750.

2. Parker, D.; Feast, W. J. Macromolecules 2001, 34, 2048-2059.

3. Holter, D.; Frey, H. Acta Polymerica 1997, 48, 298-309.

4. Read, D. J.; McLeish, T. C. B. Macromolecules 2001, 34, 1928-1945.

5. Flory, P. J. Journal of the American Chemical Society 1952, 74, 2718-2723.

6. Flory, P. J. Principles of Polymer Chemistry; Cornell University Press: Ithaca, 1953. 